\title{
Renormalization: A Number Theoretical Model
}

\section{Bertfried Fauser}

Max Planck Institute for Mathematics in the Sciences, Inselstrasse 22-26, D-04103 Leipzig, Germany. E-mail: fauser@mis.mpg.de

Received: 23 June 2006 / Accepted: 29 June 2007

Published online: 24 November 2007 - C Springer-Verlag 2007

\begin{abstract}
We analyse the Dirichlet convolution ring of arithmetic number theoretic functions. It turns out to fail to be a Hopf algebra on the diagonal, due to the lack of complete multiplicativity of the product and coproduct. A related Hopf algebra can be established, which however overcounts the diagonal. We argue that the mechanism of renormalization in quantum field theory is modelled after the same principle. Singularities hence arise as a (now continuously indexed) overcounting on the diagonals. Renormalization is given by the map from the auxiliary Hopf algebra to the weaker multiplicative structure, called Hopf gebra, rescaling the diagonals.
\end{abstract}

\section{Contents}

1. Dirichlet Convolution Ring of Arithmetic Functions . . . . . . . . . . . . 628

1.1 Definitions . . . . . . . . . . . . . . . . . . . 628

1.2 Multiplicativity versus complete multiplicativity . . . . . . . . . . . 629

1.3 Examples . . . . . . . . . . . . . . . . . . . 629

2. Products and Coproducts Related to Dirichlet Convolution . . . . . . . . . 630

2.1 Multiplicativity of the coproducts . . . . . . . . . . . . . . 632

3. Hopf Gebra: Multiplicativity Versus Complete Multiplicativity . . . . . . . 633

3.1 Plan A: The modified crossing . . . . . . . . . . . . . . . . . 633

3.2 Plan B: Unrenormalization . . . . . . . . . . . . . . . . . . 634

3.3 The co-ring structure . . . . . . . . . . . . . . . 635

3.4 Coping with overcounting : renormalization . . . . . . . . 635

4. Taming Multiplicativity . . . . . . . . . . . . . . . . . . 637

Appendix A. Some Facts about Dirichlet and Bell Series . . . . . . . . . . . . 638

A.1 Characterizations of complete multiplicativity . . . . . . . . . . 638

A.2 Groups and subgroups of Dirichlet convolution . . . . . . . . . . . . 639

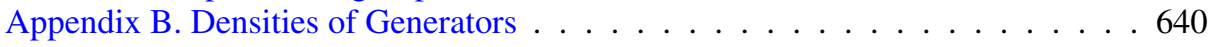

References........................ . . 640 


\section{Dirichlet Convolution Ring of Arithmetic Functions}

1.1. Definitions. In this section we recall a few well know facts about formal Dirichlet series and the associated convolution ring of Dirichlet functions [1,4]. An arithmetic function is a map $f: \mathbb{N} \rightarrow \mathbb{C}$. Equivalently we can consider integer indexed sequences of complex numbers. It is convenient to introduce formal generating functions to encode this information in a more compact form

$$
\begin{aligned}
f(s) & :=\sum_{n \geq 1} \frac{f(n)}{n^{s}}, \\
s & =\sigma+i t \in \mathbb{C},
\end{aligned}
$$

where the formal complex parameter is traditionally written as $s$. No confusion should arise between the series elements $f(n)$ and the generating function $f(s)$ formally denoted in the same way.

A ring structure is imposed in the obvious manner:

Definition 1.1. The Dirichlet convolution ring of arithmetic functions is defined on the set of arithmetic functions as

$$
\begin{aligned}
(f+g)(s) & :=\sum_{n \geq 1} \frac{f(n)+g(n)}{n^{s}}, \\
(f \star g)(s) & :=\sum_{n \geq 1} \sum_{d \mid n} \frac{f(d) \cdot g(n / d)}{n^{s}},
\end{aligned}
$$

where $\sum_{d \mid n}$ is the sum over all divisors $d$ of $n$.

The component-wise addition imposes a module structure on the arithmetic functions, and the convolution product is actually the point-wise product of the generating functions $f(s) \cdot g(s)$ as is easily seen. Furthermore the product is commutative, associative, and unital with unit $u(s):=\sum_{n \geq 1} \delta_{n, 1} n^{-s}$, where $\delta_{n, 1}$ is the Kronecker delta symbol.

If $f(1) \neq 0$, then a unique inverse Dirichlet generating function exists w.r.t. the convolution product

$$
\begin{aligned}
f \star f^{-1} & =u=f^{-1} \star f, \\
n=1: \quad f^{-1}(1) & =1 / f(1), \\
n>1: \quad f^{-1}(n) & =\frac{1}{f^{-1}(1)} \sum_{\substack{d \mid n \\
d<n}} f^{-1}\left(\frac{n}{d}\right) f(d) .
\end{aligned}
$$

The invertible arithmetic functions form a group:

$$
\begin{aligned}
f \star u & =f=u \star f, \\
(f \star g)^{-1} & =g^{-1} \star f^{-1},
\end{aligned}
$$

due to the associativity of the convolution. 
1.2. Multiplicativity versus complete multiplicativity. Two integers $n, m$ are called relatively prime if their greatest common divisor $\operatorname{gcd}(n, m)=(n, m)$ is 1 , hence if they have no prime factor in common. Many important number theoretical functions enjoy a weakened homomorphism property, called multiplicativity.

Definition 1.2. An arithmetic function $f$ is called complete multiplicative if

$$
f(n \cdot m)=f(n) \cdot f(m) \quad \forall n, m .
$$

An arithmetic function $f$ is called multiplicative if

$$
f(n \cdot m)=f(n) \cdot f(m) \quad \forall n, m \text { with }(n, m)=1 .
$$

Hence multiplicative functions fail in general to be homomorphisms of the multiplicative structure of the natural numbers iff the product has a nontrivial common prime number content $(n, m)=k$, such that $n=k \cdot n^{\prime}$ and $m=k \cdot m^{\prime}$. We may call $k$ the overlapping or meet part of $n, m$. Actually ged and lcm form a distributive lattice on the integers.

1.3. Examples. We give some examples of arithmetic functions, among them multiplicative, complete multiplicative and non-multiplicative ones, which all play important roles in number theory.

Let $n=\prod p_{i}^{r_{i}}, m=\prod p_{i}^{s_{i}}$ and define $v$ to be the function $v(n)=2^{\sum r_{i}}$. One has

$$
\begin{aligned}
v(n \cdot m) & =v\left(\prod p_{i}^{r_{i}+s_{i}}\right)=2^{\sum\left(r_{i}+s_{i}\right)}=2^{\left(\sum r_{i}\right)+\left(\sum s_{i}\right)} \\
& =2^{\sum r_{i}} 2^{\sum s_{i}}=v(n) v(m) .
\end{aligned}
$$

Hence $v$ is a homomorphism or complete multiplicative function.

The Möbius function is defined as

$$
\mu(n)=\left\{\begin{array}{cl}
1 & n=1 \\
0 & n \text { contains a square } \\
(-1)^{k} & n=\prod_{i=1}^{k} p_{i}, \quad k \text { distinct primes }
\end{array} .\right.
$$

The sequence of integer values of the Möbius function is a random-looking list of $\pm 1,0$ entries:

$$
\begin{array}{c|rrrrrrrr}
n & 1 & 2 & 3 & 4 & 5 & 6 & 7 & 8
\end{array} \ldots .
$$

Another interesting arithmetic function is the Euler totient function, which counts the number of relative prime numbers $d$ having $(d, n)=1$ smaller than $n$. Using \# for cardinality it reads $\phi=\#\{d \in \mathbb{N} ; d<n,(d, n)=1\}$ :

$$
\begin{array}{c|cccccccc}
n & 1 & 2 & 3 & 4 & 5 & 6 & 7 & 8
\end{array} \ldots .
$$

Introducing the arithmetic function $N(n)=n, \forall n$ one finds $\phi(n)=(\mu \star N)(n)=$ $n \prod_{p \mid n}\left(1-\frac{1}{p}\right)$. The Möbius and Euler totient functions are multiplicative, but not complete multiplicative. 
A further example of a non multiplicative function is the von Mangoldt function:

$$
\Lambda(n)=\left\{\begin{array}{c}
\log p \text { if } n=p^{m}, \quad m \geq 1, p \text { a prime } \\
0 \text { otherwise (including } 1 \text { ) }
\end{array}\right.
$$

Tabulated this reads

$$
\begin{array}{c|ccccccccc}
n & 1 & 2 & 3 & 4 & 5 & 6 & 7 & 8 & \ldots \\
\hline \Lambda(n) & 0 & \log 2 & \log 3 & \log 2 & \log 5 & 0 & \log 7 & \log 2 & \ldots
\end{array} .
$$

The importance of the von Mangoldt function stems from the fact that it encodes the derivation with respect to the formal parameter $s$ of a Dirichlet generating function in terms of the convolution product. We use $\sum_{d \mid n} \Lambda(d)=\log n$ to show this:

$$
\frac{\partial}{\partial s} f(s)=\sum_{n \geq 1} f(n) \frac{\partial}{\partial s} n^{-s}=\sum_{n \geq 1} f(n)(-\log n) n^{-s} .
$$

In particular one obtains for the Riemann zeta function $\zeta^{-1}=\mu, \zeta(n)=1 \forall n$ the formula

$$
-\frac{\zeta(s)^{\prime}}{\zeta(s)}=-\frac{\partial}{\partial s} \log \zeta(s)=\Lambda(s) .
$$

The von Mangoldt function appears in the Selberg formula [23], which allows one to embark on an 'elementary', that is nonanalytic, proof of the prime number theorem.

\section{Products and Coproducts Related to Dirichlet Convolution}

In previous work, we studied extensively the Dirichlet Hopf algebra of arithmetics [12]. We extract from that work the two coproducts needed for the present purpose. We dualize the (semi)ring structure ${ }^{1}$ of the natural numbers $(\mathbb{N},+, \cdot)$ using the Kronecker duality written as a scalar product $\langle\mid\rangle: \mathbb{N} \times \mathbb{N} \rightarrow \mathbb{Z}_{2},\langle n \mid m\rangle=\delta_{n, m}$.

Definition 2.3. The coproduct of addition is defined as

$$
\begin{aligned}
\Delta^{+}(n) & :=\sum_{n_{1}+n_{2}=n} n_{1} \oplus n_{2} \\
& =n_{(1)} \oplus n_{(2)},
\end{aligned}
$$

and the coproduct of multiplication is defined as

$$
\begin{aligned}
\Delta \cdot(n) & :=\sum_{n_{1} \cdot n_{2}=n} n_{1} \times n_{2}=\sum_{d \mid n} d \times \frac{n}{d} \\
& =n_{[1]} \times n_{[2]} .
\end{aligned}
$$

We introduced Sweedler indices and the Brouder-Schmitt convention [3] to denote Sweedler indices of different coproducts by different parentheses.

\footnotetext{
${ }^{1}$ We will later on always complete the natural numbers à la Grothendieck to a group, the integers, hence 'ring' will be a posteriori justified.
} 
Our coproducts allow us to define convolution algebras on the (set of coefficients of) arithmetic functions $f: \mathbb{N} \rightarrow \mathbb{C}$ together with the product $\mu$ in $\mathbb{C}$. If the codomain of such a function is in the nonnegative integers, it is an endomorphism of $\mathbb{C}$ under a suitable identification of $\mathbb{N}$ in $\mathbb{C}$. The process of extending the convolution of (set) endomorphisms on $\mathbb{N}$ to (set) homomorphisms $\operatorname{hom}(\mathbb{N}, \mathbb{C}$ ) is subtle, since ring extensions have to be considered. In our formal treatment we do not care about this.

Definition 2.4. A convolution algebra $\operatorname{Conv}(\mu, \Delta)$ is defined on homomorphisms $f$, $g \in \operatorname{hom}(\mathbb{N}, \mathbb{C})$ as

$$
\begin{aligned}
& (f+g)(n)={ }^{\prime}+{ }^{\prime}(f \times g) \Delta^{+}(n)=f(n)+g(n), \\
& (f \star g)(n)=\mu(f \times g) \Delta(n)=\sum_{d \mid n} f(n) \cdot g\left(\frac{n}{d}\right)
\end{aligned}
$$

for the addition ' + ' the product $\mu$ and coproducts $\Delta^{+}$respectively $\Delta$ '.

It is easy to show the following

Proposition 2.5. Conv $\left(+, \Delta^{+}\right)$is biassociative, biunital, bicommutative with antipode $\mathrm{S}^{+}: \mathbb{N} \rightarrow \mathbb{Z}$ given by $\mathrm{S}^{+}(n)=-n$

Note that the antipode is $\mathbb{Z}$ valued forcing us to extend the codomain of the homomorphisms at least to $\mathbb{Z}$. We introduce the Hadamard product . : $\operatorname{hom}(\mathbb{N}, \mathbb{C}) \times$ $\operatorname{hom}(\mathbb{N}, \mathbb{C}) \rightarrow \operatorname{hom}(\mathbb{N}, \mathbb{C})$, that is the coefficient-wise product of Dirichlet series, as $(f . g)(s)=\sum_{n \geq 1} f(n) \cdot g(n) n^{-1}$ to be able to state the

Proposition 2.6. $\operatorname{Conv}\left(\cdot, \Delta^{*}\right)$ is biassociative, biunital, bicommutative with antipode $\mathrm{S}^{\cdot}: \mathbb{N} \rightarrow \mathbb{Z}$ given by $\mathrm{S}^{\cdot}(n)=(N . \mu)(n)=n \cdot \mu(n)$ or alternatively written as generating function $\mathrm{S}^{*}(s)=\mu(s-1)$.

While the first statements are almost trivial, the antipode can be derived as a group inverse using a recursion argument. Tabulated it reads

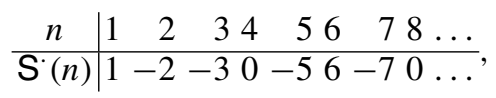

which should be compared with the table (1-9).

The coproduct of multiplication models exactly split arguments in the Dirichlet convolution. In this case the Hopf algebraic version acts directly on the elements of the series representation of the arithmetic functions. The remarkable fact is that this coproduct can be obtained from an almost trivial dualization of multiplication of integers. The coproduct of addition will come into play later. We want to make this duality explicit, using the Kronecker pairing $\langle n \mid m\rangle=\delta_{n, m}$,

$$
\begin{aligned}
\langle n+m \mid k\rangle & =\left\langle n \oplus m \mid \Delta^{+}(k)\right\rangle=\left\langle n \mid k_{(1)}\right\rangle\left\langle m \mid k_{(2)}\right\rangle \\
\Leftrightarrow \quad \Delta^{+}(k) & =\sum_{k_{1}+k_{2}=k} k_{1} \times k_{2}=k_{(1)} \oplus k_{(2)},
\end{aligned}
$$

and for the coproduct of multiplication one has

$$
\begin{aligned}
& \langle n \cdot m \mid k\rangle=\langle n \times m \mid \Delta \cdot(k)\rangle=\left\langle n \mid k_{[1]}\right\rangle\left\langle m \mid k_{[2]}\right\rangle \\
& \Leftrightarrow \quad \Delta^{\cdot}(k)=\sum_{k_{1} \cdot k_{2}=k} k_{1} \times k_{2}=\sum_{d \mid n} d \times \frac{n}{d}=k_{[1]} \times k_{[2]} .
\end{aligned}
$$


We close this discussion by exhibiting the primitive elements with respect to coaddition and comultiplication. A $(1,1)$-primitive element (or simply primitive element) $p$ is defined satisfying the relation $\Delta(p)=p \otimes 1+1 \otimes p$. Using the particular monoidal structure, i.e. direct sums for addition, cartesian product for multiplication, and the respective units for addition and multiplication, we find as an easy consequence of the definitions:

Corollary 2.7. With respect to the coproduct of addition $\Delta^{+}, 1$ is the only primitive element and $\mathbb{N}$ is additively generated by 1 .

Corollary 2.8. With respect to the coproduct of multiplication $\Delta,\left\{p_{i}\right\}_{i=1}^{\infty}$ the set of all prime numbers represents all primitive elements and $\mathbb{N}$ is multiplicatively generated by these primes.

This poses the opportunity to introduce two gradings on $\mathbb{N}$ turning the integers into a graded set, first by setting $\mathbb{N}=\oplus_{n \in \mathbb{N}} \mathbf{1}^{n}$, where every number represents its own grade. Addition is a graded map (binary 'product') under this grading. Now let $\mathbb{P}$ be the set of all prime numbers and $\mathbb{P}^{k}$ the set of all integers having exactly $k$ prime factors (including multiplicities). Let $\mathbb{P}^{0}=1$. The grading suggested by the multiplicative structures is defined as:

$$
\mathbb{N}=\oplus_{i \geq 0} \mathbb{P}^{i}
$$

This regrouping will have a great influence on how densities or the asymptotic behaviour of Dirichlet arithmetic functions have to be considered, see Appendix B. For a detailed discussion of the algebraic aspects, including Hopf algebra cohomology, see [12].

\subsection{Multiplicativity of the coproducts. A remarkable fact is the following}

Proposition 2.9. The coproduct of multiplication $\Delta^{\prime}$ is a multiplicative function.

Proof. First consider relative prime numbers $p^{r}, q^{s}$,

$$
\Delta\left(p^{r} \cdot q^{s}\right)=\sum_{d \mid p^{r} \cdot q^{s}} d \times \frac{p^{r} \cdot q^{s}}{d} .
$$

Since $p^{r} \mid q^{s}=1$, from which follows $d\left|p^{r} \cdot q^{s}=a\right| p^{r} \cdot b \mid q^{s}$, we obtain

$$
\begin{aligned}
\Delta \cdot\left(p^{r} \cdot q^{s}\right) & =\sum_{\substack{a\left|p^{r} \\
b\right| q^{s}}} a \cdot b \times \frac{p^{r} \cdot q^{s}}{a \cdot b}=\sum_{l=0}^{r} \sum_{k=0}^{s} p^{l} q^{k} \times p^{r-l} q^{s-k} \\
& =\sum_{l=0}^{r} p^{l} \times p^{r-l} \sum_{k=0}^{s} q^{k} \times q^{s-k}=\sum_{c \mid p^{r}} c \times \frac{p^{r}}{c} \sum_{d \mid q^{s}} d \times \frac{q^{s}}{d} \\
& =\Delta^{\cdot}\left(p^{r}\right) \Delta\left(q^{s}\right) .
\end{aligned}
$$

$\Delta^{\cdot}$ is not complete multiplicative due to

$$
\begin{aligned}
\Delta \cdot(4) & =1 \times 4+2 \times 2+4 \times 1 \\
\Delta(2) \Delta(2) & =(1 \times 2+2 \times 1)^{2}=1 \times 4+2 \times 2+2 \times 2+4 \times 1,
\end{aligned}
$$

which completes the proof. 
The lack of complete multiplicativity of the coproduct map spoils a major axiom of Hopf algebra theory, namely the homomorphism axiom

$$
\Delta \mu(n \times m)=(\mu \otimes \mu)(\operatorname{Id} \otimes \mathrm{SW} \otimes \operatorname{Id})(\Delta \otimes \Delta)(n \times m),
$$

which fails to hold(!) in the present case, but is only true as a multiplicative relation for $(n, m)=1$. The multiplicative convolution, despite being bicommutative, biassociative, biunital, and having a nice antipode, is alas not a Hopf algebra.

\section{Hopf Gebra: Multiplicativity Versus Complete Multiplicativity}

The fact that the convolution $\operatorname{Conv}\left(\cdot, \Delta^{*}\right)$ is not a Hopf algebra spoils the idea of employing a vast amount of standard machinery. To distinguish the presently studied antipodal convolution from a proper Hopf algebra we give it a new name.

Definition 3.10. A biassociative, biunital, antipodal convolution $\operatorname{Conv}(\mu, \Delta, \mathrm{S})$ is called a Hopf gebra (HG). If the product is a comultiplicative map and if the coproduct is a multiplicative map fulfilling Eq. (2-11) then the Hopf gebra is called multiplicative. ${ }^{2}$

3.1. Plan A: The modified crossing. To be able to deal with the multiplicative, or even the general case, one has to introduce new technical devices. For definitions etc. see $[15,10]$. A first attempt at a cure would be to ask if there could be a deformed crossing or switch $c_{V, U}: V \otimes U \rightarrow U \otimes V$ so that the homomorphism axiom Eq. (2-11) could be reestablished in a complete multiplicative fashion. This hope is nourished by the following:

Theorem 3.11. [20]: Every biassociative antipodal convolution has a unique crossing $c_{V, U}$, such that $\Delta$ is a monoid homomorphism and $\mu$ is a comonoid homomorphism

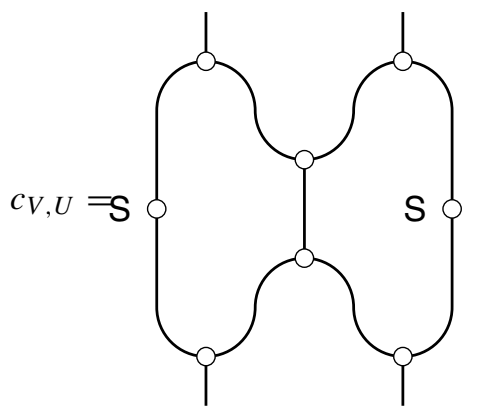

If $c_{V, U}$ is a braid, i.e. $\left(c_{V, W} \otimes I d_{U}\right)\left(I d_{V} \otimes c_{U, W}\right)\left(c_{U, V} \otimes I d_{W}\right)=\left(I d_{W} \otimes c_{U, V}\right)\left(c_{U, W} \otimes\right.$ $\left.I d_{V}\right)\left(I d_{U} \otimes c_{V, W}\right)$, on $U \otimes V \otimes W$, then the Hopf gebra is a braided Hopf algebra. If $c_{U, V}$ is a (graded) switch the Hopf gebra becomes a (graded) Hopf algebra.

The further route of such studies involves the possible classifications of crossings obtained this way, and to detect if they are braided, compute their minimal polynomial and so on. Such research is quite tedious, as was shown in [15]. The difficulties are so large that in fact plan A has to be disregarded.

\footnotetext{
2 This notion is in the Bourbaki tradition [2] and was used in [10] but originally coined by Oziewicz [20], however, with a different connotation.
} 
3.2. Plan B: Unrenormalization. We need to come up with a new strategy. The idea is to reestablish a Hopf algebra structure as close as possible to the given multiplicative Hopf gebra in question. Then use the nicely behaved Hopf algebra for computations, and try to find a transformation back to the Hopf gebra formulation. That there is actually hope to do so, stems from the fact that we are going to establish a Hopf algebra which is isomorphic to the multiplicative Hopf gebra on all relatively prime inputs and differs only on common 'overlapping' prime factors. To comply with the usage of the term 'renormalization' in physics, we need to call such a map assigning to a multiplicative Hopf gebra a Hopf algebra an 'unrenormalization' map.

Definition 3.12. The unrenormalized coproduct of multiplication $\underline{\Delta}$ related to the (renormalized) coproduct of multiplication $\Delta^{\prime}$ is recursively defined as

$$
\text { i) } \begin{aligned}
\underline{\Delta}(p) & =\Delta(p)=p \times 1+1 \times p \text { on primes } \\
\text { ii) } \underline{\Delta}(n \cdot m) & =\underline{\Delta}(n) \cdot \underline{\Delta}(m) \quad \forall n, m
\end{aligned}
$$

forcing complete multiplicativity.

In this way the homomorphism axiom (2-11) holds automatically on all pairs $n, m$ of (non-negative) integers. It is important to note that this is a minimal alteration of the coproduct in the sense that the unrenormalized coproduct differs only on the diagonal (on the gcd's) from the original coproduct. While the counit still remains as the counit of the unrenormalized coproduct, unrenormalization has, however, serious impacts, for example

Corollary 3.13. The unrenormalized antipode is given as $\underline{\mathrm{S}}(n)=(-)^{\sum r_{i}} n$, where $n=\prod_{i} p_{i}^{r_{i}}$.

This result shows that the antipode is just the grade involution with respect to the grading of the natural numbers by prime number content. This is a natural map in Hopf algebra theory, but far from being an interesting number theoretic arithmetic function, like the Möbius function, which was related to the renormalized antipode.

We can now wonder which duality connects multiplication and the new unrenormalized co-product.

Corollary 3.14. Let $n=\prod_{i} p_{i}^{r_{i}}$ and $m=\prod_{j} p_{j}^{s_{j}}$. The pairing ( | ) defined by

$$
(n \mid m)=\prod_{i} \delta_{r_{i}, s_{i}} r_{i} !=z_{n}
$$

dualizes the multiplication $\cdot$ into $\underline{\Delta}$.

Proof. (Sketch). Use Laplace expansion demanding that · and $\underline{\Delta}$ are Milnor-Moore dual w.r.t. ( | ). For details see [12].

Note that also in this case all alterations are just scalings: $(n \mid m)=z_{n}\langle n \mid m\rangle$, which is up to a rescaling by $z_{n}$, the Kronecker delta again. 
3.3. The co-ring structure. Before we try to set up the number theoretical model of renormalization, we want to exhibit the co-ring structure. This implies a relation between the coproduct of addition and the coproduct of multiplication in analogy with a ring structure. Such relations were used in [14] to investigate new group branchings.

Let us introduce a further group-like coproduct $\delta: \mathbb{P} \rightarrow \mathbb{P} \times \mathbb{P}, \delta(p)=p \times p$. Coaddition and comultiplication are related as $\left(n=\prod_{i} p_{i}^{r_{i}}\right)$

$$
\begin{aligned}
\Delta \cdot(n) & =\sum_{d \mid n} d \times \frac{n}{d} \\
& =\delta^{\Delta^{+}}(n)=\prod_{i}\left(p_{i} \times p_{i}\right)^{\Delta^{+}\left(r_{i}\right)} \\
& =\sum_{r_{i}^{\prime}+r_{i}^{\prime \prime}=r_{i}}\left(\prod_{i} p_{i}^{r_{i}^{\prime}}\right) \times\left(\prod_{i} p_{i}^{r_{i}^{\prime \prime}}\right),
\end{aligned}
$$

where the notion $\Delta^{\cdot}=\delta^{\Delta^{+}}$should be taken as a mnemonic only.

The unrenormalized case follows along the same lines, and actually can be used to define the unrenormalized coproduct of addition:

$$
\underline{\Delta}^{+}(n):=\sum_{n_{1}+n_{2}=n}\left(\begin{array}{c}
n \\
n_{1}
\end{array}\right) n_{1} \times n_{2} .
$$

Let $n=\prod_{i} p_{i}^{r_{i}}$, unrenormalized addition and unrenormalized multiplication relate as:

$$
\begin{aligned}
\underline{\Delta}^{\prime}(n) & =\delta \underline{\Delta}^{+}(n) \\
& =\sum_{r_{i}^{\prime}+r_{i}^{\prime}=r_{i}}\left(\begin{array}{c}
r_{i}^{\prime}+r_{i}^{\prime} \prime \\
r_{i}
\end{array}\right) \prod_{i} p_{i}^{r_{i}^{\prime}} \times \prod_{i} p_{i}^{r_{i}^{\prime \prime}} .
\end{aligned}
$$

The appearance of the binomial factors is well known from calculations in quantum field theories, describing the coproduct of scalar fields for example.

3.4. Coping with overcounting : renormalization. Our paradigm is that the number theoretically interesting structure is the renormalized one, which is only multiplicative, and hence forms a multiplicative Hopf gebra (HG) only. To use nice algebraic machinery, we associate to it an unrenormalized Hopf algebra (HA) which differs only on common prime content, hence in a minimal way. The relation of the HG and HA can be summarized in the following commutative diagram:

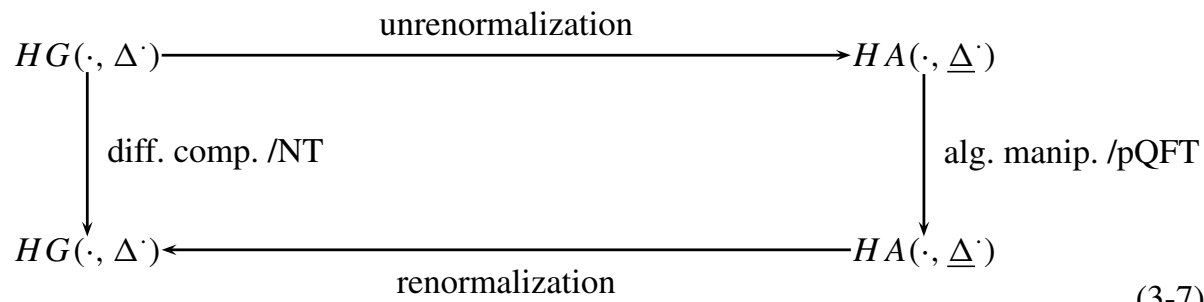


Number theoretical (NT) computations in the Dirichlet ring of arithmetic functions, i.e. in the convolution ring over the Hopf gebra (HG), are performed along the left down arrow and are usually involved and complex. Perturbative quantum field theory (pQFT) starts with a Hopf algebra structure assuming an algebra structure on the duals of the fields, either explicitly or implicitly. Then algebraic calculations are performed explicitly or implicitly using the underlying Hopf algebra (HA) structure. However, the final formal expressions are plagued by infinities, which are removed by a rescaling technique called renormalization. Our point is that this rescaling ends up in a Hopf gebra in analogy to number theory. The first step, the unrenormalization, is not seen in physics, since the modelling is done by assuming a Hopf algebra structure or equivalently a compatible algebra structure of the fields and their duals, which vice versa implies a comultiplication. The technique of renormalization hence copes with overcounting on the diagonals (gcd generalized to common maximal ideals). In pQFT these overcountings are infinite, since summations are replaced by integrations which in general diverge. In number theory one obtains finite overcountings, and a Hopf algebra approach would have just failed to work by producing wrong results. However, after having established the relation of the diagram Eq. (3-7) one attempted to try to unrenormalize problems in number theory and to use methods from QFT to handle them and 'renormalize' the formal result. Our approach opens at least two new possibilities:

a: pQFT starts with a HA structure, the unrenormalization is hence superfluous. Due to scalings by counterterms renormalization takes care of 'overcounting the diagonal'. An enlargement of modelling to start with unrenormalized quantities would possibly allow to introduce number theoretic machinery, i.e. celebrated theorems and particular techniques, to solve problems in physics.

b: Via the unrenormalization, there may arise new possibilities to deal with hard number theoretic problems in the ring of arithmetic functions by using methods from quantum field theory.

Hence renormalization should be understood as a sort of rewriting rule, allowing insights to be moved from one side to the other.

There are several approaches to the theory of renormalization, discussed for example in the topical review [7]. However, from our point of view, the approach proposed by Brouder-Schmitt [3], based on Epstein-Glaser renormalization [8], seems to be more natural and we have adopted it in our work [12]. Therein it was shown for the example of occupation number representations that the ordering process which we introduced in [9] also applies for QM and used both algebraic structures, the unrenormalized and renormalized ones. Since the same process of deformation, but on another level of complexity, produces the renormalization mechanism, we argue that the 'ordering' or 'deformation' if done on the higher level of complexity -multiplication versus addition, or composition versus multiplication- enters at least in a twofold manner, the more complex one giving rise to the renormalization map. In terms of symmetric functions this leads to the Hopf algebra of plethysm [13]. The crucial fact is that addition can be obtained as iteration of the successor map, multiplication as the iteration of the addition, and exponentiation as the iteration of multiplication. Further generalization fails, since the iteration functor needs a transposition, which is equivalent to demanding a commutative binary underlying operation [17]. In that sense, our number theoretic model needs to be enlarged to include exponentiation to actually parallel the 'renormalization' encountered in pQFT. 


\section{Taming Multiplicativity}

A complete multiplicative function $g$ is defined if its values are known on all primes, i.e. on $\mathbb{P}$. Let $n=\prod_{i} p_{i}^{r_{i}}$, then complete multiplicativity allows to write

$$
g(n)=g\left(\prod_{i} p_{i}^{r_{i}}\right)=\prod_{i} g\left(p_{i}\right)^{r_{i}} .
$$

However, a multiplicative function $f$ needs to be specified on all prime powers $\left\{p_{i}^{k}\right\}$, $\forall i, k$

$$
f(n)=f\left(\prod_{i} p_{i}^{r_{i}}\right)=\prod_{i} f\left(p_{i}^{r_{i}}\right) .
$$

While both sets have the same cardinality it is awkward that a multiplicative function is not well defined by its values on generators, here the primes in $\mathbb{P}$.

In what follows, we want show how one might tame multiplicativity by giving data only on primes, and controlling the values on $f\left(p^{n}\right)$ by a recursion involving a complete multiplicative function. This idea is based on the analogy that the expectation values of powers of quantum fields $\left\langle 0\left|(\psi(x))^{2}\right| 0\right\rangle$ should be computable from a function of the expectation values $\langle 0|\psi(x) \psi(y)| 0\rangle$ in a suitable limit $y \rightarrow x$.

The device we want to use is that of Bell series. These are series encoding an arithmetic function on all prime powers of a given prime $p$.

Definition 4.15. A Bell series of an arithmetic function $f$ for a fixed prime $p$ is given as an ordinary power series

$$
f_{p}(x)=\sum_{n \geq 0} f\left(p^{n}\right) x^{n}
$$

employing a formal indeterminate $x$.

Corollary 4.16. If $f$ is complete multiplicative its Bell series reads

$$
f_{p}(x)=\sum f(p)^{n} x^{n}=\frac{1}{1-f(p) x} .
$$

The Bell series of the Möbius function and the Euler totient function read

$$
\begin{aligned}
\mu_{p}(x) & =1-x, \\
\phi_{p}(x) & =\frac{1-x}{1-p x},
\end{aligned}
$$

showing that they are not complete multiplicative. The most important fact about Bell series for us is that the Dirichlet convolution product of arithmetic functions is transformed into the Cauchy product of Bell series. Let $h=f \star g$, then

$$
h_{p}(x)=f_{p}(x) g_{p}(x),
$$

reducing the complexity of the operation dramatically. 
We use an example from Apostol [1] to demonstrate how this might be used to model the process of renormalization in number theory in analogy to renormalization in pQFT, by adding counterterms or modifying the pole structure of the 'propagator'.

Let $g$ be complete multiplicative, and recall that then $g(1)=1$. We define a recursion for a multiplicative function $f$ so that all values of $f$ on prime powers are determined. In terms of coefficients a particular recursion reads

$$
f\left(p^{n+1}\right)=f(p) f\left(p^{n}\right)-g(p) f\left(p^{n-1}\right) .
$$

This allows to compute the Bell series

$$
f_{p}(x)=\frac{1}{1-f(p) x+g(p) x^{2}} .
$$

It can be shown that Eq. (4-8) follows from Eq. (4-7) and vice versa. Using this recursion, it is now possible to establish the following product formula:

$$
f(m \cdot n)=f(m) f(n)-\sum_{\substack{d \mid \operatorname{gcd}(n, m) \\ 1<d \leq \operatorname{gcd}(n, m)}} g(d) f\left(\frac{m \cdot n}{d^{2}}\right) .
$$

Together with Eq. (4-8) this establishes a number theoretic analog of renormalization theory. $g(p)$ would serve as an additive renormalization of the 'propagator' $\frac{1}{1-f(p) x+g(p) x^{2}}$ and the sum in the right hand side of Eq. (4-9) constitutes counterterms.

Acknowledgements. It is a pleasure to thank Peter Jarvis for many helpful discussions and for ongoing collaboration on this subject. Part of this work was done in Hobart during a visit supported by the ARC research grant DP0208808, and the Alexander von Humboldt Foundation.

\section{Appendix A. Some Facts about Dirichlet and Bell Series}

A.1. Characterizations of complete multiplicativity. Since multiplicativity versus complete multiplicativity plays a major role in our argumentation we want to recall useful characterizations of multiplicativity.

Lambek [16] proved that an arithmetical function $f$ is completely multiplicative iff its Hadamard product distributes over every Dirichlet product:

$$
f .(g \star h)=(f . g) \star(f . h)
$$

for all arithmetical functions $g, h$. In terms of coefficients this reads

$$
f(n) \sum_{d \mid n} g(d) h(n / d)=\sum_{d \mid n} f(d) g(d) f(n / d) h(n / d) .
$$

This can be rephrased saying that the convolution is a Laplace pairing [11] for the Hadamard product. Carlitz [5] posed the problem to characterize complete multiplicativity by distributivity over particular Dirichlet convolutions. Let $\tau=\zeta \star \zeta$ be the number of positive divisors function. $f$ is complete multiplicative iff

$$
f . \tau=(f . \zeta) \star(f . \zeta)=f \star f .
$$

A nice way to generalize such notions is by using Möbius categories $\mathcal{C}[18,19]$. These are categories defined to generalize and unify the theory of Möbius inversions. In terms 
of morphisms one investigates incidence functions $f, g$ forming an incidence algebra $A(\mathcal{C})$ by employing the product

$$
(f \star g)(\alpha)=\sum_{\alpha^{\prime} \alpha^{\prime \prime}=\alpha} f\left(\alpha^{\prime}\right) g\left(\alpha^{\prime \prime}\right) .
$$

An incidence function is complete multiplicative iff

$$
f(\alpha)=f\left(\alpha^{\prime}\right) f\left(\alpha^{\prime \prime}\right)
$$

with $\alpha=\alpha^{\prime} \alpha^{\prime \prime}$ the composition of morphisms. Now, Lambek's characterization generalizes to this case, while Carlitz' characterization has to be altered [22]. It is, however, nice to have a generalization to this general setting allowing to export the concept of multiplicativity to incidence (or functionals on operator) algebras. This way of generalization is needed on the way to establish our analogy between number theory and pQFT in a more concrete way.

A.2. Groups and subgroups of Dirichlet convolution. This section follows the exposition of Dehaye [6]. Let $\mathrm{F}_{0}$ be the set of multiplicative functions different from the zero function $\mathbf{0}(n)=0$ for all $n$. This amounts to have $f(1)=1$ for all $f$ in $\mathbf{F}_{0}$. The pair $\left(\mathrm{F}_{0}, \star\right)$ is an abelian group with Dirichlet convolution as product.

For any prime $p$ we define $\mathbf{F}^{p}=\left\{f \in \mathrm{F}_{0} \mid f(n)=0\right.$ for every $n$ s.t. $\left.p \nmid n\right\}$. That is, an $f \in \mathrm{F}^{p}$ has support on prime powers $p^{k}$ only. For every prime $p,\left(\mathrm{~F}^{p}, \star\right)$ is a subgroup of $\mathrm{F}_{0}$. Furthermore, there exists an isomorphism between $\mathrm{F}^{p}$ and the group of upper-triangular non-zero infinite matrices $\mathbf{M}^{1}$,

$$
\mathbf{M}^{1}=\{m \in \mathbf{M} \mid m(a, a)=1, \forall a \in \mathbb{N} \text {, and } m(a, b)=0, \forall a, b \in \mathbb{N} \text { s.t. } a>b\} .
$$

$\mathbf{M}^{1}$ is a group with the infinite unit matrix as identity element. The isomorphism $\phi$ : $\mathrm{F}^{p} \rightarrow \mathbf{M}^{1}$ is given by

$$
\phi(f)=\left(\begin{array}{ccccc}
1 & f(p) & f\left(p^{2}\right) & f\left(p^{3}\right) & \cdots \\
0 & 1 & f(p) & f\left(p^{2}\right) & \cdots \\
0 & 0 & 1 & f(p) & \cdots \\
0 & 0 & 0 & 1 & \cdots \\
\vdots & \vdots & \vdots & \vdots & \ddots
\end{array}\right)
$$

and

$$
\phi(f) \cdot \phi(g)=\phi(f \star g),
$$

where in the 1.h.s. the product is matrix multiplication. This isomorphism shows that the Bell series are particular Dirichlet series or restrictions of Dirichlet series to the subgroup $\mathrm{F}^{p}$. These groups are isomorphic for every pair of primes $p_{i}, p_{j}$.

It is possible to consider $F_{0}$ as a complete (or Cartesian) direct product of the subgroups $\mathrm{F}^{p}$ for all primes

$$
\mathrm{F}_{0}=\bar{\prod}_{i \in \mathbb{N}} \mathrm{F}^{p_{i}}
$$

It can be shown [6] that 
a) The group $F_{0}$ is torsion free (i.e. has no element of finite order).

b) $\mathrm{F}^{p}$ is a subgroup of $\mathrm{F}_{0}$ for every prime $p$, all such subgroups are pairwise isomorphic and are isomorphic to infinite upper-triangular non-zero matrices or to Bell series.

c) $\mathrm{F}_{0}$ is isomorphic to the complete direct product of the subgroups $\mathrm{F}^{p}$.

d) $\mathrm{F}_{0}$ is divisible and has a natural structure of a vector space over $\mathbb{Q}$.

\section{Appendix B. Densities of Generators}

We want to emphasise another point which connects our work with renormalization of quantum fields. As our discussion here and in [12] demonstrated, one can grade the natural numbers in two canonical ways attached to addition and multiplication. If we generate the natural numbers additively, we have but one generator, the one 1 , which is the target of the successor map, and all numbers are generated as successors of the zero 0 . The successor map is assumed to have no torsion and composition is associative. From this construction it is evident that the density of natural numbers in the natural numbers $d\left(n, n_{0}\right)$ with respect to the 1 as generator is constant. That is, in every neighbourhood, that is an interval containing $n_{0}$, of a natural number $n_{0}$, one finds the same density of natural numbers.

The second way to grade the natural numbers was induced by the multiplicative structure and the primitive elements, i.e. generators, were shown to be the set of prime numbers $\left\{p_{i}\right\}$. However, the density of prime numbers in the natural numbers is a nontrivial function. The celebrated prime number theorem states that the number of prime

numbers below $n_{0}$ is $\frac{n_{0}}{\log n_{0}}$ for $n_{0} \rightarrow \infty$. This renders it obvious that a multiplicative construction of the integers behave quite differently with respect to the densities of generators in the natural numbers. A. Petermann showed in a remarkable paper [21] that a renormalization group analysis provides a proof for the prime number theorem. This supports our claim that the present simplified model of renormalization is actually rich enough to contain main features of renormalization in quantum field theory.

It is possible to iterate this process by asking what kind of 'primitive elements' occur if one looks for exponentiation as an iteration of multiplication. This question leads into the realm of modular forms and one obtains higher order corrections in the densities of 'higher primitive elements' along the same lines as one obtains higher order loop corrections, and divergencies in perturbative quantum field theory. This will be explored elsewhere.

\section{References}

1. Apostol, T.M.: Introduction to Analytic Number Theory. New York: Springer-Verlag, 1979 [fouth printing 1995]

2. Bourbaki, N.: Elements of Mathematics: Algebra I Chapters 1-3. Berlin: Springer-Verlag, 1989

3. Brouder, C., Schmitt, W.: Renormalization as a functor on bialgebra. J. Pure Appl. Alg. 209, 477-495 (2007)

4. Brüdern, J.: Einfuihrung in die analytische Zahlentheorie. Berlin: Springer-Verlag, 1995

5. Carlitz, L.: Problem E 2268. Amer. Math. Monthly 78, 1140 (1971)

6. Dehaye, P.-O.: On the structure of the group of multiplicative arithmetical functions. Bull. Belg. Math. Soc. Simon Stevin 9(1), 15-21 (2002)

7. Ebrahimi-Fard, K., Kreimer, D.: The Hopf algebra approach to Feynman diagram calculations. J. Phys. A: Math. Gen. 38, R385-R407 (2005)

8. Epstein, H., Glaser, V.: The role of locality in perturbation theory. Ann. Inst. Henri Poincaré 19, 211-295 (1973) 
9. Fauser, B.: On the Hopf-algebraic origin of Wick normal-ordering. J. Phys. A: Math. Gen. 34, 105-115 (2001)

10. Fauser, B.: A Treatise on Quantum Clifford Algebras. Konstanz, 2002, Habilitationsschrift, available at http://arxiv.org/list/math.QA/0202059, 2002

11. Fauser, B., Jarvis, P.D.: A Hopf laboratory for symmetric functiuons. J. Phys. A: Math. Gen. 37(5), 1633-1663 (2004)

12. Fauser, B., Jarvis, P.D.: The Dirichlet Hopf algebra of arithmetics. J. Knot Theor. and Its Ramif. 16(4), 379-438 (2007)

13. Fauser, B., Jarvis, P.D.: The Hopf algebra of plethysms. Work in progress, 2007

14. Fauser, B., Jarvis, P.D., King, R.C., Wybourne, B.G.: New branching rules induced by plethysm. J. Phys A: Math. Gen. 39, 2611-2655 (2006)

15. Fauser, B., Oziewicz, Z.: Clifford Hopf gebra for two dimensional space. Misc. Alg. 2(1), 31-42 (2001)

16. Lambek, J.: Arithmetical functions and distributivity. Amer. Math. Monthly 73, 969-973 (1966)

17. Lawvere, F.W., Rosebrugh, R.: Sets for Mathematics. Cambridge: Cambridge Univ. Press, 2003

18. Leroux, P.: Les Catégories Möbius. Cahiers Top. Géom. Différ. Catég. 16, 280-282 (1975)

19. Leroux, P.: Reduced matrices and $q$-log-concavity properties of $q$-Stirling numbers. J. Combin. Theory Ser. A 54, 64-84 (1990)

20. Oziewicz, Z.: Clifford Hopf gebra and biuniversal Hopf gebra. Czech. J. Phys. 47(12), 1267-1274 (1997)

21. Petermann, A.: The so-called Renormalization Group method applied to the specific prime numbers logarithmic decrease Eur. Phys. J. C 17, 367-369 (2000)

22. Schwab, E.D.: Characterizations of Lambek-Carlitz type. Arch. Math. (Brno) 40, 295-300 (2004)

23. Selberg, A.: An elementary proof of the prime number theorem. Ann. Math. 50, 305-313 (1949)

Communicated by A. Connes 